\title{
Insufferably Dilly-Dallying and Lazy Editorial (IDLE) Syndrome: Proposed Diagnostic Criteria for Identifying Slothful Journal Editors
}

\author{
Scott O. Lilienfeld ${ }^{1,2}$ and Robert D. Latzman ${ }^{3}$ \\ ${ }^{1}$ Emory University, Atlanta, GA, USA \\ ${ }^{2}$ University of Melbourne, Parkville, VIC, Australia \\ ${ }^{2}$ Georgia State University, Atlanta, GA, USA \\ E-mail: slilien@emory.edu and rlatzman@gsu.edu
}

\begin{abstract}
Numerous authors have bemoaned the myriad shortcomings of the journal peer review process (McCook, 2006; Peters \& Ceci, 1982). Among the most frequently voiced complaints are the apparent arbitrariness of editorial decisions; biases of reviewers; unclear or inconsistent reviewer or editorial feedback; and unduly long delays between initial journal submissions and editorial decisions (Berquist, 2016; Stahel \& Moore, 2014; Ware, 2007). Accumulating evidence suggests that much of the variance in author dissatisfaction with peer review stems from an underlying constellation of covarying editorial behaviors that have been variously characterized by aggrieved authors as idleness, indolence, slothfulness, dilatoriness, torpidity, languor, inertia, or more colloquially, laziness (see also Baumeister, 1992). Nevertheless, to our knowledge, no formal means of identifying this vexing syndrome of editorial behaviors is available in the published literature. The absence of standardized diagnostic criteria for this distinctive behavioral pattern is problematic given ample circumstantial evidence of its deleterious impact on author well-being, including intense distress, demoralization, and intermittent homicidal urges.
\end{abstract}

In response to this glaring gap, the authors (a psychology journal editor and psychology/psychiatry journal associate editor, respectively) ${ }^{1}$ hereby present a proposed new entry to the next edition of the American

\footnotetext{
${ }^{1}$ Vehement assertions by some of their academic colleagues (and numerous authors who have been recipients of their action letters) notwithstanding, neither of the authors meets the diagnostic criteria for IDLE to the best of their knowledge. Then again, unpublished research by the first author suggests that between 95 and 100 percent of journal
}

Psychiatric Association's Diagnostic and Statistical Manual (DSM), which we tentatively term Insufferably Dilly-Dallying and Lazy Editorial (IDLE) syndrome. IDLE syndrome will of course require further investigation before it is enshrined in the formal psychiatric nomenclature, although we believe that it is ready for inclusion in Section III ("Emerging Measures and Models") of the DSM (American Psychiatric Association, 2013) as well for potential consideration in the forthcoming edition of the International Classification of Diseases (ICD-11). We believe that this provisional diagnostic criterion set should prove useful in facilitating research on this widely recognized yet surprisingly understudied (Dean \& Forray, 2019) syndrome. The proposed criteria for IDLE syndrome, derived by the authors using a rigorous process of gossiping, griping, moaning-groaning, and kvetching with numerous academic colleagues across a twodecade span, supplemented by the time-honored statistical technique of "eyeball factor analysis" (McCall, 1994) conducted with the aid of an ample supply of ethyl alcohol, are presented below. We welcome suggested additions or revisions to this criterion set from interested or irate readers of The Clinical Psychologist. ${ }^{2}$

\section{Diagnostic Criteria for Insufferably Dilly-Dallying and Lazy Editorial (IDLE) Syndrome}

editors who meet criteria for IDLE are convinced that they do not have the disorder.

${ }^{2}$ Complaint letters by outraged journal editors should, however, be sent to The Clinical Psychologist editorial staff rather than to the authors. 
A. A consistent pattern of indecisiveness or reluctance/unwillingness to provide with clear or informative feedback, as indicated by 4 or more of the following:

(1) Recurrent failure to desk-reject (triage) manuscripts that are clearly inappropriate

for the journal or inadequate in quality, leading authors to wait needlessly for several months for rejection letters

(2) Repeatedly sitting on manuscripts for 1 month or more before finally desk-rejecting them, often with unclear or inadequate justification

(3) Extremely brief (e.g., 1-2 sentence) action letters that provide authors with little or no guidance for how to address reviewer comments (e.g., "Please address the reviewers' comments below. We look forward to receiving your resubmission")

(4) Recurrent failure to provide guidance to authors when reviewer comments are inconsistent or mutually exclusive (e.g., Reviewer 1 writes "The Discussion section is much too brief" and Reviewer 2 writes "The Discussion section is much too lengthy," and the editor writes "Please address the reviewers' concerns regarding the length of the Discussion section")

(5) Repeatedly providing inconsistent feedback to authors (e.g., asking them to add an analysis and later asking them to remove it) across multiple rounds of revision, or repeatedly changing one's mind about whether the manuscript is publishable

(6) Using identical "boilerplate" language for all action letters (e.g. "Although the current manuscript has a number of strengths, the reviewers noted a number of weaknesses that preclude publication of the manuscript its current form")

(7) Encouraging authors to repeatedly revise manuscripts even when the odds of acceptance are extremely low, resulting in numerous rejections of manuscripts that should have been rejected earlier

B. A consistent pattern of pathological dependency on reviewer input, reluctance/unwillingness to evaluate manuscripts independently, or failure to even inspect manuscripts before acting on them as indicated by 4 or more of the following:
(1) Recurrent tendency to send manuscripts to reviewers who have little or no expertise in the subject matter of the paper, often based on a casual perusal of authors and co-authors listed in manuscript reference sections

(2) Excessive reliance on reviewers' recommendations, as indicated by repeated editorial statements such as "Reviewer A is asking more changes," "Reviewer B is still not convinced by your arguments," or "I cannot accept the manuscript until all reviewers are satisfied with it"

(3) Recurrent failure to read submitted manuscripts or to at best to skim them, as indicated by editorial comments that contradict statements in the manuscript (e.g., "Please address the limitations of your design" when the authors explicitly included a limitations section," "You did not conduct analysis $\mathrm{X}$ in your manuscript" when analysis $X$ was reported in the submitted supplemental materials)

(4) Recurrent tendency to send manuscripts back to reviewers over and over again even when requested changes are extremely minimal

(5) Recurrent tendency to send revised manuscripts back to the same reviewers 2 or more times out of reluctance or unwillingness to appraise manuscripts independently

(6) Recurrent failure to act on manuscripts for 1 or more months even when all reviewer recommendations have been submitted

(7) Recurrent tendency to accept manuscripts only when all reviewer recommendations are positive, leading to rejection of almost or all controversial and/or innovative manuscripts

C. Not attributable to generalized laziness, indecisiveness, low motivation, inattentiveness, sleep disorders, or grandiose narcissism.

D. Produces clinically significant distress and/or impairment in submitting authors, as indicated by recurrent complaints to editors that their manuscripts have been handled ineptly; recurrent angry emails (which may be obscene) or threats of physical harm from authors; incessant whining and moaning to faculty colleagues, postdocs, and students; and marked increases in depressive, anxiety, insomnia, dissociative, substance use disorder, and anger-related symptoms. 
E. Duration of at least 6 months or more. Journal editors exhibiting the features of IDLE syndrome for more than one week but less than 6 months should be diagnosed with Acute Insufferably Dilly-Dallying and Lazy Editorial Syndrome.

\section{Diagnostic Specifiers:}

(a) With versus Without Email Nonresponsiveness; Delusions

(b) With versus Without Omniscient

\section{Risk Factors, Associated Features, and Differential Diagnosis}

Several studies suggest an elevated prevalence of IDLE syndrome in academic departments that provide few or no incentives for editorial activities, as well as among professional organizations and publishers that provide little guidance to or supervision of editors. IDLE syndrome is sometimes associated with excessive devotion to non-editorial activities, such as publishing 20 or more peer-reviewed papers per year or submitting 10 or more federal grant proposals per year. Anecdotal reports suggest that being extremely slow to return manuscripts reviews, as well as a tendency to turn down virtually all manuscripts review requests, are risk factors for IDLE syndrome among reviewers who later become journal editors. Although IDLE syndrome tends to generate intense distress among authors, some researchers maintain that this syndrome is associated with heightened levels of academic success among editors themselves, many of whom have considerably more time to devote to publication and grant activity than do other editors.

Circumstantial evidence indicates that IDLE syndrome is comorbid with several conditions, including generalized laziness, chronic self-absorption, absentmindedness, passive-aggressive personality disorder, sadistic personality disorder, and Editorial God Complex, but it should be distinguished from each of them. In rare circumstances, IDLE syndrome may co-exist with both sadistic personality disorder and Editorial God Complex (a constellation sometimes referred to by scholars as the Editor From Hell Dark Triad), in which cases it may pose a dire threat to author mental health, not to mention journal publication lags.

\section{Prevalence and Demographic Differences}

The precise prevalence of IDLE syndrome is unknown, although informal reports suggest that it afflicts between 5 and 10 percent of journal editors and perhaps a comparable proportion of associate editors. IDLE syndrome appears to be especially prevalent among editors of journals that receive extremely high numbers of submissions. One study points to an elevated prevalence of IDLE syndrome among editors of predatory journals. There are no known gender, age, or race differences in IDLE syndrome; similarly, there are no published cross-cultural studies of IDLE syndrome.

\section{Development and Course}

IDLE syndrome tends to be associated with an early onset and usually emerges during the affected individuals' first journal editorship. One longitudinal study suggests that a history of early manuscript rejections is a risk factor for later IDLE syndrome, which may reflect a relatively advanced defense mechanism against the painful trauma associated with this early rejection. Although there are few systematic data on the course of IDLE syndrome, numerous reports strongly suggest that the condition is often chronic, even life-long. Full remission appears to be rare. In approximately 5 to 10 percent of cases, IDLE syndrome apparently worsens across successive journal editorships, occasionally resulting in Editorial Decision Paralysis Syndrome (marked by a complete breakdown in all vital, lower-level editorial functions, accompanied by a failure to make any editorial decisions at all). Editors diagnosed with IDLE syndrome for one journal typically meet criteria for IDLE syndrome for subsequent journals they edit.

\section{Potential Consequences}

The morbidity and mortality associated with IDLE syndrome are unknown, although an elevated frequency of emailed and phoned-in death threats sent to editors with IDLE syndrome compared with other editors has been reported in several studies. These death threats have almost always ceased promptly upon manuscript acceptance.

IDLE syndrome is associated with heightened levels of trichotillomania - not among journal editors themselves, that is, but among authors who have been observed to pull their hair out following multiple IDLE 
syndrome-related behaviors. Intermittent explosive disorder has also been reported as an occasional outcome among journal author victims. Scattered anecdotal reports further indicate that IDLE syndrome is associated with a variety of other symptoms among authors, including telephone scatologia; repeatedly muttering the journal name in vain under one's breath during faculty meetings; and marked increases in vocal tics and motor tics (especially coprolalia and copropraxia, respectively) whenever they encounter the journal name. Some investigators have also reported delusions of persecution among author victims of IDLE syndrome, although in many cases this apparent symptom may reflect accurate perceptions of persecution by sadistic journal editors.

\section{Treatment}

Observational studies suggest that IDLE syndrome is difficult or impossible to treat, at least using currently available interventions. In most cases, IDLE syndrome is associated with poor insight and low motivation for treatment. Although numerous authors have enthusiastically advocated prefrontal lobotomy or other forms of psychosurgery for editors with IDLE syndrome, there are no solid data, aside from subjective reports from authors of pleasurable fantasies (which may be highly cathartic; Personal Communication from Several Thousand Authors, 2019), supporting such practices. With regard to author victims of IDLE syndrome, data from several case studies raise the possibility that primal scream therapy can be an effective short-term intervention, as can extortion of journal editors (see "Potential Consequences").

\section{Conclusions}

IDLE syndrome is widely discussed by journal authors, especially when editors are not present in the room. Nevertheless, this disorder has been the subject of relatively little systematic research, largely because no formal method of diagnosing it has heretofore been available. We hope that these proposed diagnostic criteria for IDLE syndrome will provide a helpful impetus for future investigation regarding the pathophysiology of this poorly understood, presently untreatable, and enormously annoying condition. Promising approaches to the treatment and prevention of IDLE syndrome are urgently needed as a means of addressing the ailing mental health of the rapidly growing but underserved population of journal authors.

\section{References}

American Psychiatric Association (2013). Diagnostic and statistical manual of mental disorders, fifth edition (DSM-5). Washington, D.C.: Author.

Baumeister, R. F. (1992). Dear journal editor, it's me again. Simulation \& Gaming, 23, 219-221.

Berquist, T.H. (2017). Peer review: Is the process broken? American Journal of Roentgenology, 209, 1-2.

Dean, K. L., \& Forray, J. M. (2019).

Reopening the black box of editorship: Who reviews journal editors? Journal of Management Education, 43, 3-9.

McCall, M. (1994). Identifying leadership potential in future international executives: Developing a concept. Consulting Psychology Journal: Practice and Research, 46, 49-63.

McCook, A. (2006). Is peer review broken? Submissions are up, reviewers are overtaxed, and authors are lodging complaint after complaint about the process at top-tier journals. What's wrong with peer review? The Scientist, 20(2), 26-35.

Peters, D.P. \& Ceci, S.J. (1982). Peer-review practices of psychological journals: The fate of published articles, submitted again. Behavioral and Brain Sciences, 5, 187-255.

Stahel, P. F., \& Moore, E. E. (2014). Peer review for biomedical publications: We can improve the system. BMC Medicine, 12(1), 179.

Ware M. (2007). Peer review: Benefits, perceptions and alternatives. London: Publishing Research Consortium. 